\title{
Infrared spectroscopy with multivariate analysis to interrogate endometrial tissue: a novel and objective diagnostic approach
}

\author{
SE Taylor, I,2, KT Cheung', II Patel', J Trevisan', HF Stringfellow', KM Ashton ${ }^{2}$, NJ Wood ${ }^{2}$, PJ Keating ${ }^{2}$, \\ PL Martin-Hirsch ${ }^{1,2}$ and FL Martin ${ }^{*, 1}$
}

'Centre for Biophotonics, Lancaster Environment Centre, Lancaster University, Bailrigg, Lancaster LAI 4YQ, UK; ' ${ }^{2}$ ancashire Teaching Hospitals NHS Trust, Royal Preston Hospital, Sharoe Green Lane North, Fulwood, Preston, Lancashire PR2 9HT, UK

BACKGROUND: Endometrial cancer is the most common gynaecological malignancy in the United Kingdom. Diagnosis currently involves subjective expert interpretation of highly processed tissue, primarily using microscopy. Previous work has shown that infrared (IR) spectroscopy can be used to distinguish between benign and malignant cells in a variety of tissue types.

METHODS: Tissue was obtained from 76 patients undergoing hysterectomy, 36 had endometrial cancer. Slivers of endometrial tissue (tumour and tumour-adjacent tissue if present) were dissected and placed in fixative solution. Before analysis, tissues were thinly sliced, washed, mounted on low-E slides and desiccated; I IR spectra were obtained per slice by attenuated total reflection Fouriertransform IR (ATR-FTIR) spectroscopy. Derived data was subjected to principal component analysis followed by linear discriminant analysis. Post-spectroscopy analyses, tissue sections were haematoxylin and eosin-stained to provide histological verification.

RESULTS: Using this approach, it is possible to distinguish benign from malignant endometrial tissue, and various subtypes of both. Cluster vector plots of benign (verified post-spectroscopy to be free of identifiable pathology) vs malignant tissue indicate the importance of the lipid and secondary protein structure (Amide I and Amide II) regions of the spectrum.

CONCLUSION: These findings point towards the possibility of a simple objective test for endometrial cancer using ATR-FTIR spectroscopy. This would facilitate earlier diagnosis and so reduce the morbidity and mortality associated with this disease. British Journal of Cancer (201 I) 1 04, 790-797. doi:I0.1038/sj.bjc.6606094 www.bjcancer.com

Published online 15 February 20II

(C) 2011 Cancer Research UK

Keywords: attenuated total reflection Fourier-transform infrared spectroscopy; endometrial cancer; endometrium; infrared spectroscopy; multivariate analysis

Attenuated total reflection Fourier-transform infrared (ATR-FTIR) spectroscopy allows the objective classification of biological material on a molecular level. Previous studies have shown the potential of vibrational spectroscopy in categorising cancer and intraepithelial neoplasia in tissues such as the cervix, prostate, and various parts of the gastrointestinal tract (Sindhuphak et al, 2003; German et al, 2006; Maziak et al, 2007; Walsh et al, 2008; Baker et al, 2009; Sahu et al, 2010). Many of these tissues are classified and analysed as either normal or abnormal. In contrast, human endometrium is a tissue that exhibits biological plasticity, capable of adopting several different histological conformations depending on the hormonal milieu.

Endometrial cancer is the most common gynaecological malignancy in the United Kingdom. This is most likely to be related to the increasing prevalence of obesity among women (Renehan et al, 2008). Adipose tissue contains the enzyme aromatase, responsible for the conversion of the adrenal-derived androgen androstenedione to oestrone, an oestrogen. This acts unopposed by progesterone to stimulate endometrial proliferation

*Correspondence: Dr SE Taylor; E-mail: siantaylor@gmail.com or Dr FL Martin; E-mail: f.martin@lancaster.ac.uk

Revised 03 December 2010; accepted 22 December 2010; published online I5 February 201 I in perimenopausal and postmenopausal women (Schindler, 2009). Oestrone also appears to exert genotoxic effects (Yared et al, 2002). Endometrial cancer is frequently underestimated as a fairly benign condition. Indeed, early, low-grade disease has an excellent prognosis; however, a significant minority of patients are less fortunate and have advanced or high-grade disease (Uharcek, 2008).

Endometrial cancers are classified according to their behaviour and histological appearance into two groups. Endometrioid cancers are often referred to as type I disease. They are associated with obesity, polycystic ovarian syndrome, unopposed oestrogen hormone replacement therapy, and the perimenopausal age group. All of these factors contribute to a relative oestrogen excess (Bokhman, 1983). Endometrioid cancers are graded 1-3 according to the proportion of solid tissue within the tumour, with grade 3 containing the least glandular tissue and being the most aggressive (Horn et al, 2007). Type II endometrial cancers include the uterine serous and clear cell subtypes. These are found in older postmenopausal women, are unconnected with oestrogen, and behave in a more aggressive manner. Carcinosarcomas are frequently included in this category; however, their true relation to other uterine cancer subtypes is uncertain (D'Angelo and Prat, 2010). Type I and II endometrial cancers have different mutation profiles and precursor lesions (Sherman, 2000; Fadare and Zheng, 2008). 
Biomolecules absorb energy in the mid-infrared (IR) region of the electromagnetic spectrum (Martin et al, 2010). Attenuated total reflection Fourier-transform IR spectroscopy exploits this property, measuring the absorbance of IR within this region. Each individual chemical bond has its own vibrational characteristics, causing it to absorb IR at a particular frequency (usually expressed as wavenumber $=1$ /wavelength). Plotting wavenumber against intensity of absorbance generates a spectrum that represents the quantity and type of bonds present within the material examined. When applied to biological material this distinctive pattern is known as the 'biochemical-cell fingerprint'. Changes in the type, conformation, or quantity of bonds generate different spectra; this could be used to differentiate between benign and malignant tissues. Powerful computational data reduction and separation techniques are required to interpret the vast quantity of data generated (Martin et al, 2007). Here, principal component analysis (PCA) followed by linear discriminant analysis (LDA) has been used. Principal component analysis identifies variance and compresses the data. Linear discriminant analysis demonstrates separation between different categories such as histological types. Combined together, these two techniques reveal the chemical bonds that are most important in such separation.

In previous work, transmission FTIR microspectroscopy was applied using a synchrotron source to de-waxed paraffinembedded endometrial tissue (Kelly et al, 2009). For endometrial spectroscopy to have a practical application, it must be simple and function without the need for a particle accelerator. Attenuated total reflection Fourier-transform IR spectroscopy fulfils these requirements. This is the first description of ATR-FTIR spectroscopy in endometrial tissue and addresses the potential applications of this technique in the future.

\section{PATIENTS AND METHODS}

\section{Study participants}

Informed consent to take endometrial tissue for use in research was obtained from women undergoing hysterectomy. Ethical committee approval was obtained (LREC no. 05/Q1302/83; Preston, Chorley and South Ribble Ethical Committee). Patients were allocated study numbers based on their preoperative diagnostic category. In total, 101 endometrial samples were obtained from 76 patients, 36 of whom had endometrial cancer (see Table 1). The indications for hysterectomy in the benign group included bleeding problems (dysfunctional uterine bleeding, $n=6$ ), pelvic mass (fibroids, $n=6$; ovarian mass, $n=7$ ), pelvic pain (endometriosis, $n=4$; adenomyosis, $n=6$; endometriosis and adenomyosis, $n=2$ ), premalignant disease (cervical intraepithelial neoplasia, $n=2$; suspected hyperplasia, $n=2)$, and prophylaxis (previous breast cancer, $n=5$ ).

\section{Tissue preparation}

After surgical resection, unfixed hysterectomy specimens were transported directly to the pathology laboratory. Here, a consultant histopathologist dissected out a sliver of endometrial tissue. If the uterus contained tumour, a piece of this was sampled together with a piece of adjacent macroscopically benign endometrium (if present); otherwise, a representative piece of endometrium was chosen. Tissue samples were then placed in Surepath cytology medium. The time from removal of the uterus from the patient to placement of the sample in fixative was $<20$ min.

All samples were stored in Surepath at room temperature for between $48 \mathrm{~h}$ and 4 weeks. After fixation, tissue samples were thinly sliced ( $\leqslant 1-\mathrm{mm}$ thick/slice) by hand using a Stadie-Riggs
Table I Hierarchical classification system and subgroup numbers as spectra, samples, and patients

\begin{tabular}{|c|c|c|c|}
\hline Tissue classification & Spectra (n) & Samples (n) & Patients (n) \\
\hline \multicolumn{4}{|l|}{ Normal } \\
\hline \multicolumn{4}{|l|}{ Premenopausal } \\
\hline Proliferative & 250 & 25 & 13 \\
\hline Early secretory & 80 & 8 & 4 \\
\hline Mid secretory & 20 & 2 & I \\
\hline Late secretory & 60 & 6 & 3 \\
\hline Menstrual & 61 & 6 & 3 \\
\hline On progesterone & 50 & 5 & 3 \\
\hline \multicolumn{4}{|l|}{ Postmenopausal } \\
\hline Atrophic & 219 & 22 & | | \\
\hline Other & 40 & 4 & 2 \\
\hline \multicolumn{4}{|l|}{ Cancer } \\
\hline \multicolumn{4}{|l|}{ Endometrioid } \\
\hline Grade I & 179 & 18 & 9 \\
\hline Grade 2 & 140 & 14 & 7 \\
\hline Grade 3 & 140 & 14 & 7 \\
\hline \multicolumn{4}{|l|}{ Non-endometrioid } \\
\hline Uterine serous & 60 & 6 & 3 \\
\hline Clear cell & 20 & 2 & I \\
\hline Mixed & 40 & 4 & 2 \\
\hline Carcinosarcoma & 80 & 8 & 4 \\
\hline Adenosarcoma & 40 & 4 & 2 \\
\hline \multicolumn{4}{|l|}{ Tumour adjacent } \\
\hline \multicolumn{4}{|l|}{ Endometrioid (adjacent) } \\
\hline Atrophic & 240 & 24 & 12 \\
\hline Hyperplasia & 80 & 8 & 4 \\
\hline \multicolumn{4}{|c|}{ Non-endometrioid (adjacent) } \\
\hline Atrophic & 200 & 20 & 10 \\
\hline Hyperplasia & 0 & 0 & 0 \\
\hline
\end{tabular}

Abbreviation: $n=$ number

platform and Thomas blade (Martin and McLean, 1995). Two slices were prepared from each endometrial specimen. The tissue samples were orientated so that the first slice consisted of the surface of the endometrium and the second, the tissue immediately below. Each slice was washed in three separate $10-\mathrm{ml}$ volumes of distilled $\mathrm{H}_{2} \mathrm{O}$. Slices were mounted on low-E reflective glass slides and placed in a desiccator for a minimum of $48 \mathrm{~h}$. An overview of the methodology is shown in Figure 1.

\section{Attenuated total reflection Fourier-transform infrared spectroscopy}

Infrared spectra were obtained using a Bruker Vector 22 FTIR spectrometer with Helios ATR attachment containing a diamond crystal (Bruker Optics Ltd, Coventry, UK); 10 spectra were acquired per slide, each from a different area of the tissue slice. The ATR crystal was cleaned with dry tissue paper between each spectra acquisition, and a background reading was taken prior to each new slide. A cut-off value for the Amide I peak was designated as 0.12 , to ensure spectral quality (Trevisan et al, 2010). Spectra were cut to between 1800 and $900 \mathrm{~cm}^{-1}$, baseline corrected and normalised to Amide I $\left(1650 \mathrm{~cm}^{-1}\right)$. Because of the non-destructive nature of this bio-analytical approach, the post-spectroscopy sections were then stained with haematoxylin and eosin (H\&E). Macroscopically normal endometrium sometimes contains important lesions (intraepithelial carcinoma, subtle areas of hyperplasia, and carcinoma restricted to the endometrium) identifiable under the microscope. It is not possible to totally validate a tissue as normal or benign; however, this allowed histological categorisation and verification of the tissue sections analysed in this study. 


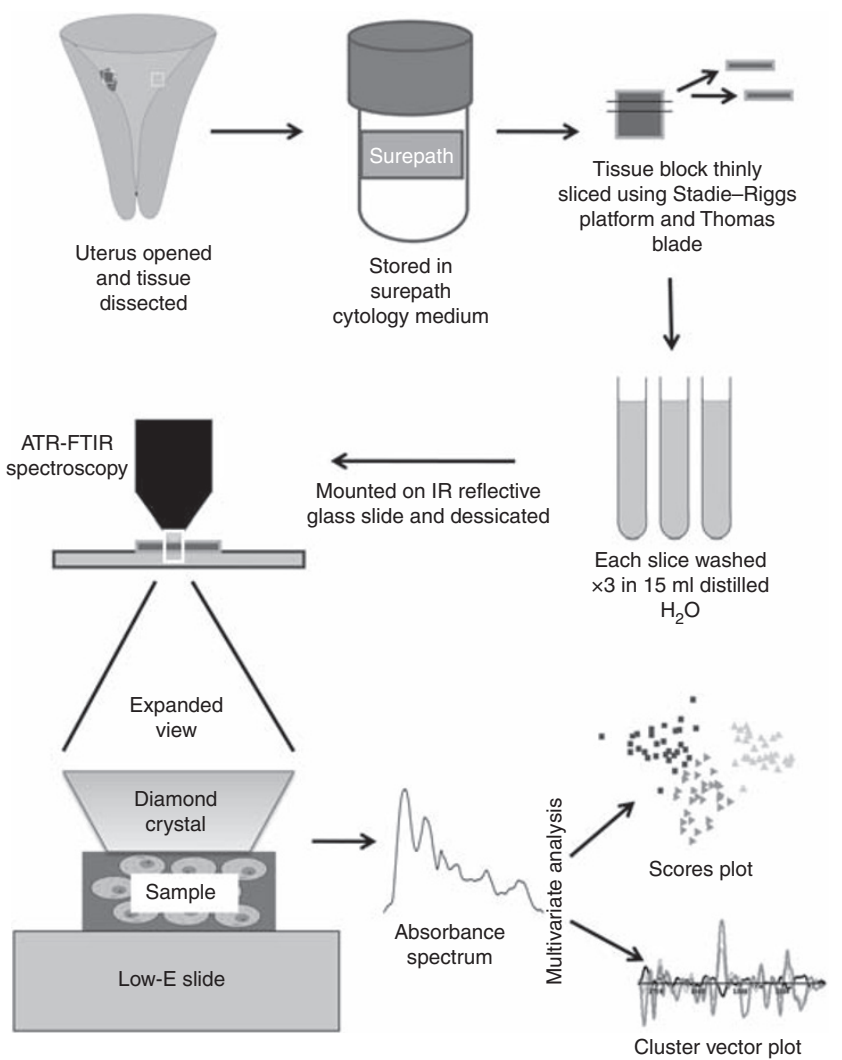

Figure I Diagrammatic representation of methodology.

\section{Computational analysis}

Multivariate analysis was performed with PCA followed by LDA using MATLAB R2008a software (The Mathworks Inc., Natick, MA, USA). Principal component analysis was used for preliminary data reduction and to demonstrate variance in an unsupervised manner. Linear discriminant analysis derives vectors from the principal components (PCs) and so minimises the within-category differences (which would mostly be associated with typical heterogeneity in any tissue sample) while maximising betweencategory discriminating characteristics (i.e., those most likely to be diagnostic). This allows separation between groups to be visualised more clearly. Multivariate analysis results are visualised in two different ways: scores plots and cluster vector plots. Scores plots are scatter charts drawn using the data values obtained after PCALDA (i.e., the 'scores') as Cartesian coordinates. Cluster vector plots are 'pseudo-spectra' (in the sense that as spectra, they contain values per wavenumber, although they contain coefficients of linear combinations) obtained by the following construction: each cluster vector is associated with a different histology type, it corresponds exactly to the vector that points from the mean spectrum of a reference histology type to mean spectrum from its specific histology type (Martin et al, 2007; Llabjani et al, 2010). This construction is done inside the vector space spanned after the PCA - LDA data reduction. These techniques were applied to total spectra per slide. All wavenumbers are rounded down to the nearest whole number.

\section{RESULTS}

Principal component analysis-LDA of the biochemicalcell-fingerprint region $\left(1800-900 \mathrm{~cm}^{-1}\right)$ was used to derive scores plots and corresponding cluster vector plots for several combinations of tissue types. Principal component analysis-LDA scores
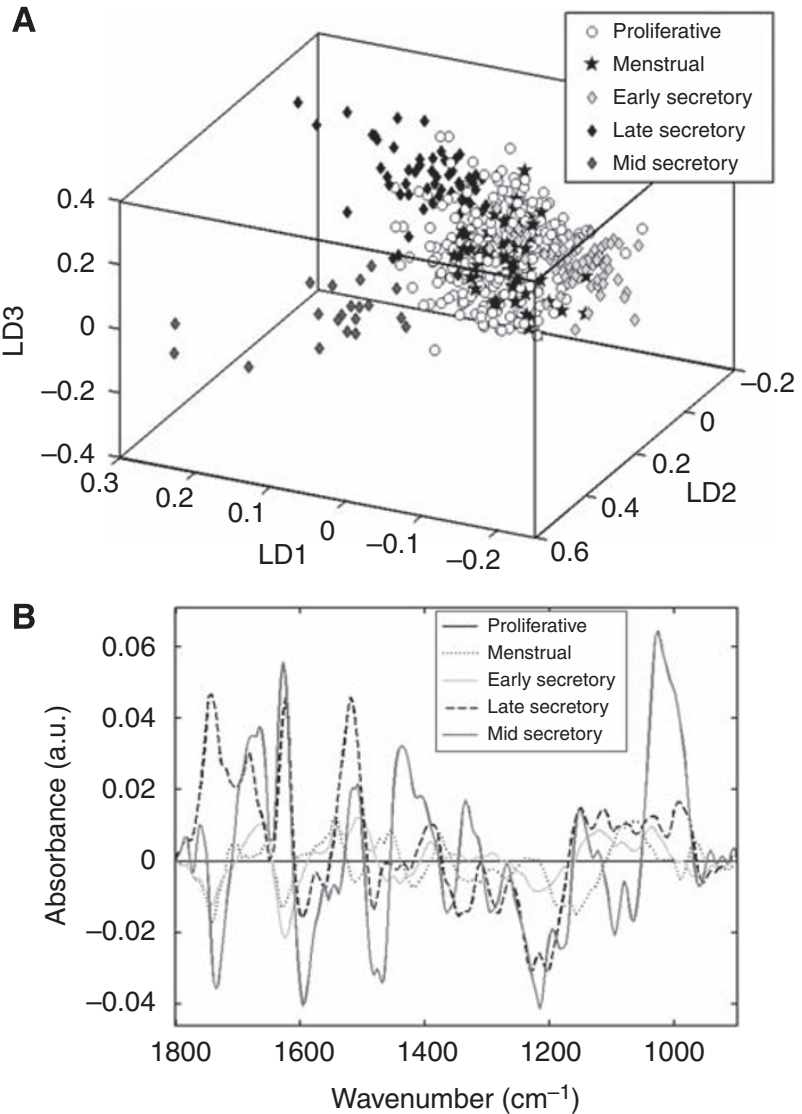

Figure 2 Comparison of normal premenopausal endometrial tissue subtypes through the menstrual cycle. (A) Rotated scores plot. (B) Cluster vector plot (proliferative tissue as comparator).

plots display each spectrum as a point in multidimensional space (number of dimensions is determined by $n-1$, where $n$ is the number of groups being compared) with proximity implying spectral, and therefore biochemical, similarity. The cluster vector plots also display variance, but here it is shown as the difference between one group (plotted as the baseline) as compared with all other groups at all wavenumbers.

\section{Tracking normal tissue through the menstrual cycle}

Normal endometrium, obtained from premenopausal women, was classified by the histological phase of the menstrual cycle. Clustering within and separation between most phases was observed. This was most clearly visible after rotation of the three-dimensional (3D) plot (Figure 2A). Clear separation is seen between the three subgroups of secretory endometrium. Proliferative phase endometrium and menstrual phase endometrium did not separate. The cluster vector plot shown in Figure 2B uses proliferative endometrium as the baseline and all others are compared with this. The phases adjacent to the proliferative phase in the cycle (menstrual and early secretory) are the most biochemically similar to it. Late secretory endometrium exhibits large peaks in the lipid $\left(1743 \mathrm{~cm}^{-1}\right)$, Amide I $\left(1624 \mathrm{~cm}^{-1}\right)$, Amide II $\left(1519 \mathrm{~cm}^{-1}\right)$, and asymmetric phosphate stretching vibrations $\left(v_{\mathrm{as}} \mathrm{PO}_{2}^{-}\left(1203 \mathrm{~cm}^{-1}\right)\right)$ regions. Mid-secretory endometrium also has prominent peaks in the lipid region $\left(1735 \mathrm{~cm}^{-1}\right)$, Amide I $\left(1666,1627,1597 \mathrm{~cm}^{-1}\right)$, Amide II $\left(1516 \mathrm{~cm}^{-1}\right)$, other protein regions $\left(1481,1435 \mathrm{~cm}^{-1}\right), v_{\text {as }} \mathrm{PO}_{2}^{-}\left(1215 \mathrm{~cm}^{-1}\right)$, and symmetric phosphate stretching vibrations $\left(v_{\mathrm{s}} \mathrm{PO}_{2}^{-}\left(1095 \mathrm{~cm}^{-1}\right)\right)$ regions. The largest peak is at $1026 \mathrm{~cm}^{-1}$; this corresponds with glycogen, 
a biochemical substrate known to be present at high levels in midsecretory endometrium (Robles et al, 1972).

\section{Benign tissue $v s$ cancer}

Benign and malignant endometrial tissues were compared. The benign group consisted of tissues from all the women recruited to the study who did not have endometrial cancer. After spectroscopy analyses, these tissue sections were H\&E-stained; in all cases, the designated tumour sections contained cytological atypia and altered tissue architectural characteristics, whereas the designated benign sections appeared normal, although postmenopausal tissues typically showed an absence of glandular elements. The tumour tissues were subdivided into endometrioid cancer and non-endometrioid cancer. Figure $3 \mathrm{~A}$ shows the scores plot for these three categories. There is a degree of overlap between them, but $\sim 80 \%$ of separation between benign and malignant spectra can be achieved by drawing a line perpendicular to LD1, near the point of origin. Figure $3 \mathrm{~B}$ shows the cluster vectors of the two malignant classes $v s$ benign tissue. The majority of the difference between benign and malignant tissue lies in the lipid $\left(1735 \mathrm{~cm}^{-1}\right)$ and Amide I/II $\left(1624,1570,1516 \mathrm{~cm}^{-1}\right)$ regions of the spectra, although there are also important contributions from a protein band $\left(1477 \mathrm{~cm}^{-1}\right), v_{\text {as }} \mathrm{PO}_{2}^{-}\left(1230 \mathrm{~cm}^{-1}\right)$, RNA/carbohydrate $\left(1168 \mathrm{~cm}^{-1}\right)$, and phosphorylated proteins $\left(968 \mathrm{~cm}^{-1}\right)$.

Endometrioid cancers and non-endometrioid cancers are spectrally similar between 1500 and $900 \mathrm{~cm}^{-1}$ as the cluster vector plots exhibit similar patterns, although several peaks are shifted by
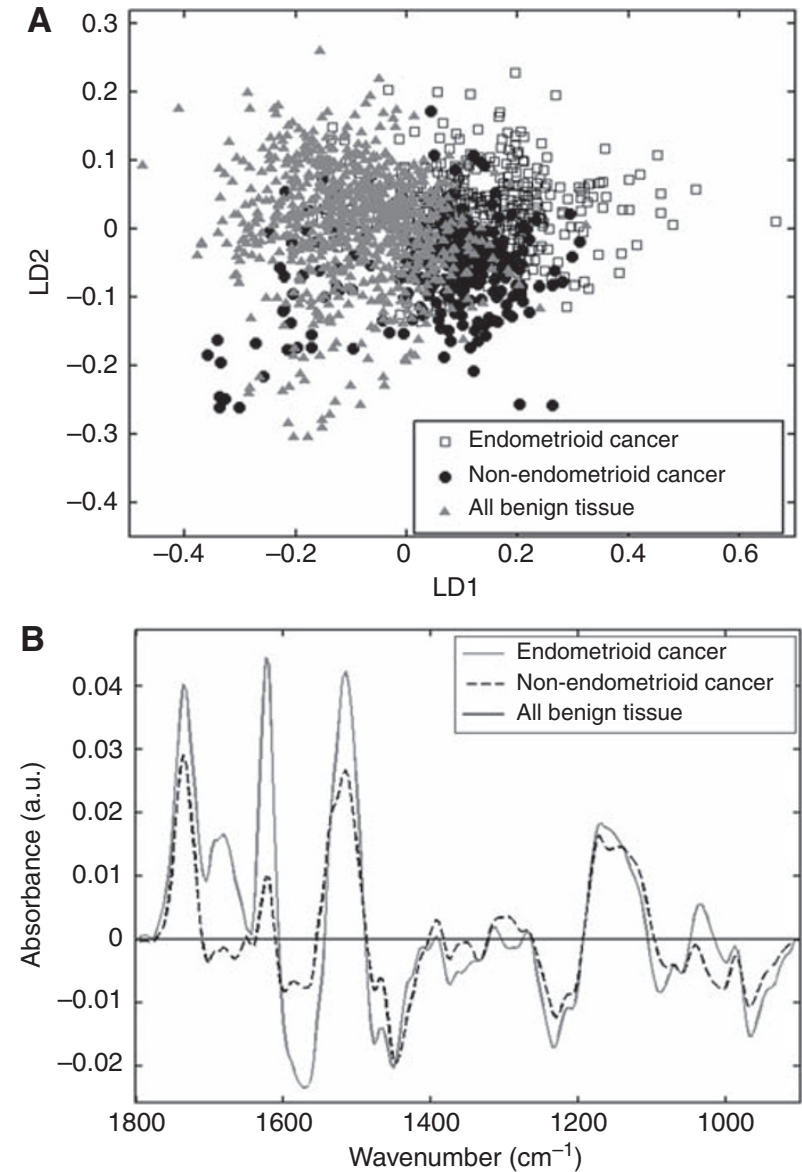

Figure 3 Comparison of malignant subtypes (endometrioid and nonendometrioid) with benign endometrial tissue. (A) Scores plot. (B) Cluster vector plot (benign tissue as comparator). a few wavenumbers. Both have peaks at around $1450 \mathrm{~cm}^{-1}$, with further peaks at $1230 \mathrm{~cm}^{-1}\left(v_{\mathrm{as}} \mathrm{PO}_{2}^{-}\right)$and $1168 \mathrm{~cm}^{-1}$ (RNA/ carbohydrate). Between 1800 and $1500 \mathrm{~cm}^{-1}$, the vectors differ in magnitude of absorbance, with larger lipid $\left(1735 \mathrm{~cm}^{-1}\right)$, Amide I $\left(1624 \mathrm{~cm}^{-1}\right)$ and Amide II $\left(1570,1516 \mathrm{~cm}^{-1}\right)$ peaks together with a separate peak at $1681 \mathrm{~cm}^{-1}$ (Amide I) for endometrioid cancers (see Table 2 for a full list of distinguishing wavenumbers).

\section{Benign tissue $v s$ tumour and tumour-adjacent tissue}

Proliferative endometrial tissue was compared with tumour tissue and its corresponding tumour-adjacent macroscopically normal tissue (verified as such following microscopic examination of post-spectroscopy H\&E-stained sections). Figure 4A and C show endometrioid cancer compared with corresponding tumouradjacent tissue compared with proliferative tissue for cluster segregation in the scores plot and discriminating features in the loadings plot. Likewise, Figure $4 \mathrm{~B}$ and $\mathrm{D}$ show non-endometrioid cancer compared with corresponding tumour-adjacent tissue compared with proliferative tissue. The proliferative tissue spectra used for comparison were the same in both. On histological examination, the tumour-adjacent tissue was benign and atrophic in the vast majority of cases (see Table 1 ).

Figure 4A shows clustering of all three groups and separation between endometrioid cancer and tumour-adjacent tissue. Here, the points (representing spectra) from proliferative tissue overlap with the other two categories, predominantly overlying the tumour-adjacent tissue plus a small area where all three categories are present. In contrast, Figure $4 \mathrm{~B}$ shows less clear separation between the tumour and tumour-adjacent spectra. On close inspection, there appears to be two clusters within the nonendometrioid cancer category. The smaller of these groups is mingled with the tumour-adjacent and proliferative points and consists of spectra from two patients with uterine serous carcinoma. In such pathology, normal-looking cells under microscopy are likely to be at least partially tumourigenic; this approach in the future might even determine the tumourigenic potential of adjacent cell populations.

Table 2 Distinguishing wavenumbers $\left(\mathrm{cm}^{-1}\right)$ of endometrioid and nonendometrioid cancers vs normal benign endometrium with likely originating bond-type

\begin{tabular}{|c|c|c|}
\hline $\begin{array}{l}\text { Endometrioid } \\
\text { cancer }\left(\mathrm{cm}^{-1}\right)\end{array}$ & $\begin{array}{l}\text { Non-endometrioid } \\
\text { cancer }\left(\mathrm{cm}^{-1}\right)\end{array}$ & $\begin{array}{l}\text { Tentative } \\
\text { assignment }\end{array}$ \\
\hline $\begin{array}{l}1736 \\
1682\end{array}$ & 1736 & $\begin{array}{l}\text { Lipid } \\
\text { Amide I }\end{array}$ \\
\hline \multirow[t]{2}{*}{1624} & 1624 & Amide I \\
\hline & $160 \mid$ & Amide I \\
\hline 1570 & 1570 & Amide II \\
\hline 1516 & 1516 & Amide II \\
\hline 1477 & 1477 & ?Protein \\
\hline \multirow[t]{2}{*}{1450} & & ?Protein \\
\hline & | 447 & ?Protein \\
\hline 1373 & & $\begin{array}{l}\text { COO-symmetric } \\
\text { stretching }\end{array}$ \\
\hline \multirow[t]{2}{*}{1234} & & $v_{\mathrm{as}} \mathrm{PO}_{2}^{-}$ \\
\hline & $\begin{array}{l}1231 \\
1173\end{array}$ & $\begin{array}{l}\mathrm{V}_{\mathrm{as}} \mathrm{PO}_{2}^{-} \\
\text {Carbydrate }\end{array}$ \\
\hline \multirow[t]{2}{*}{1169} & & Carbohydrate \\
\hline & 1142 & $\mathrm{C}-\mathrm{O}$ stretch (nu CO) \\
\hline \multirow{5}{*}{$\begin{array}{l}1088 \\
1061 \\
1034\end{array}$} & & $v_{s} \mathrm{PO}_{2}^{-}$ \\
\hline & |061 & $v_{\mathrm{s}} \mathrm{PO}_{2}^{-}$ \\
\hline & & Glycogen \\
\hline & 1003 & Glycogen \\
\hline & 968 & Protein phosphorylation \\
\hline 964 & & Protein phosphorylation \\
\hline
\end{tabular}

$?=$ possibly 

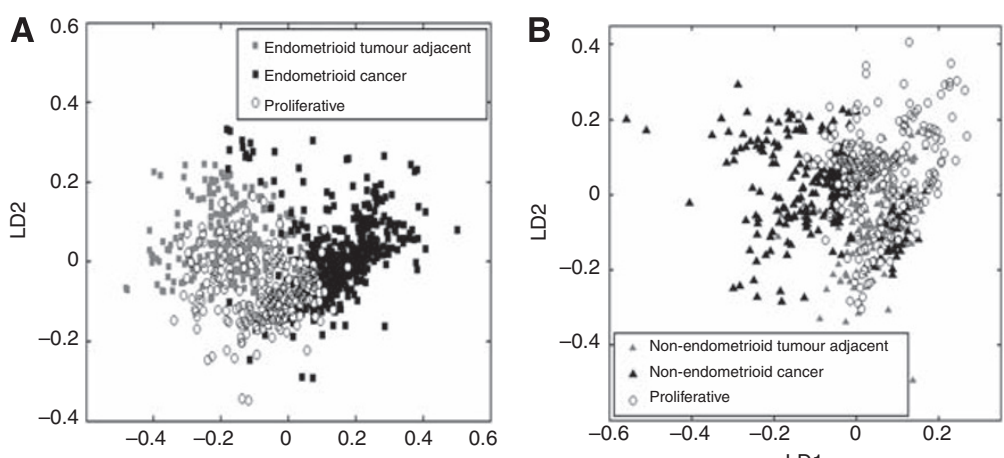

LD1
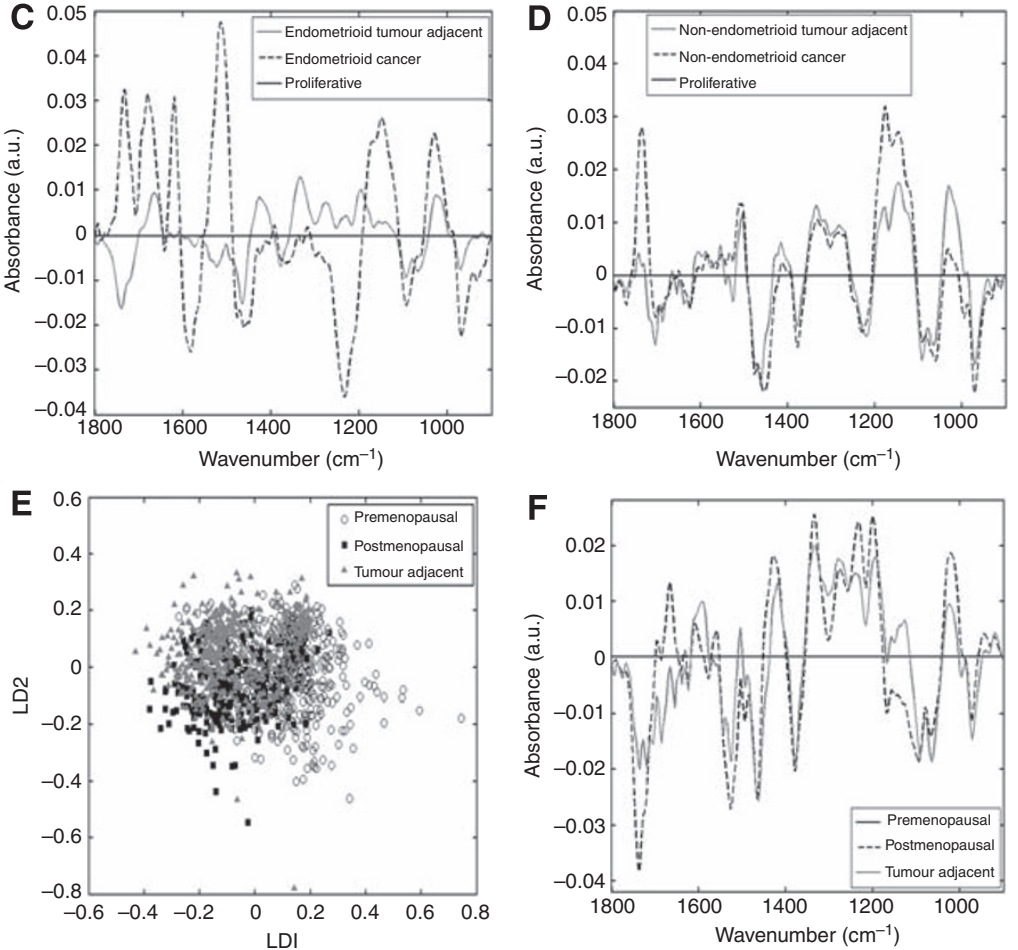

Figure 4 Comparison of cancer tissue with corresponding tumour-adjacent tissue and normal proliferative endometrium. (A) Endometrioid scores plot. (B) Non-endometrioid scores plot. (C) Endometrioid cluster vector plot (proliferative as comparator). (D) Non-endometrioid cluster vector plot (proliferative as comparator). (E) All benign tissue scores plot. (F) All benign tissue cluster vector plot (premenopausal as comparator).

Figure $4 \mathrm{C}$ is the cluster vector plot derived from Figure 4A, comparing endometrioid cancer and tumour-adjacent tissue against proliferative endometrium. The most important wavenumbers in distinguishing between proliferative endometrium and endometrioid cancer are $1732 \mathrm{~cm}^{-1}$ (lipid), $1681 / 1620 \mathrm{~cm}^{-1}$ (both Amide I), 1581/ $1516 \mathrm{~cm}^{-1}$ (both Amide II), $1234 \mathrm{~cm}^{-1}\left(v_{\mathrm{as}} \mathrm{PO}_{2}^{-}\right), 1149 \mathrm{~cm}^{-1}$ (carbohydrate), $1020 \mathrm{~cm}^{-1}$ (glycogen), and $968 \mathrm{~cm}^{-1}$ (protein phosphorylation). The differences between proliferative endometrium and endometrioid tumour-adjacent tissue are much less pronounced.

In Figure $4 \mathrm{D}$, the cluster vectors of non-endometrioid tumour-adjacent and cancer tissue are compared with normal proliferative endometrium. Unlike endometrioid cancer and its tumour-adjacent tissues, these cluster vectors are near-identical, representing similarity between non-endometrioid cancer and its corresponding adjacent tissue. The non-endometrioid cancer plot demonstrates a more pronounced single peak at $1736 \mathrm{~cm}^{-1}$ (lipid region) and a double peak at 1177 and $1146 \mathrm{~cm}^{-1}$ (?carbohydrate). The tumour-adjacent tissue has a larger peak at $1030 \mathrm{~cm}^{-1}$ (glycogen).

To strengthen the validity of our findings, all benign tissue (divided into premenopausal and postmenopausal tissue, all subtypes) was compared with all tumour-adjacent tissue as another category; such an examination would lend insight into the integrity of our tissue sampling and verify our post-spectroscopy histological analysis of the tissue sections. For instance, one would reasonably expect parameters such as myometrial invasion, blood vessel invasion, and cervical involvement to give rise to a marked number of outliers in the tumour-adjacent tissue spectral points in the scores plot. The scores plot in Figure 4E illustrates the large degree of overlap between the three groups in the absence of such outliers (particularly so in the tumour-adjacent tissue cluster), with PCA - LDA essentially unable to separate them. The premenopausal cluster exhibits the only degree of separation and a small number of outliers, which is something one might reasonably expect as a characteristic of this category. The cluster vector plot in Figure 4F shows only small differences between the tumouradjacent and postmenopausal tissues. The most significant of these is within the lipid band with a smaller amplitude negative peak at $1735 \mathrm{~cm}^{-1}$ for tumour-adjacent tissue than for postmenopausal tissue, whose peak was slightly shifted to $1739 \mathrm{~cm}^{-1}$. As the majority of tumours studied were from postmenopausal women, this similarity suggests that the tumour-adjacent tissue is predominantly biochemically normal or benign. 

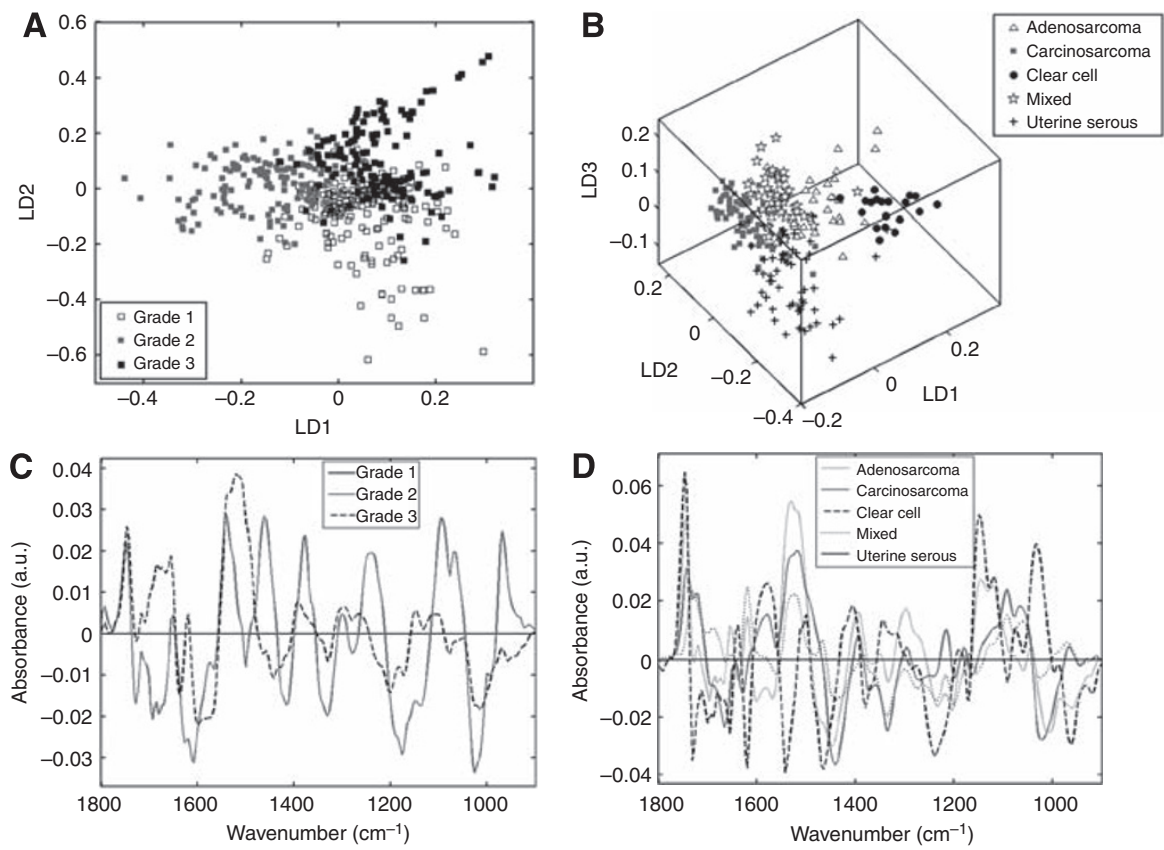

Figure 5 Comparison of grades of endometrioid carcinoma and subtypes of non-endometrioid carcinoma. (A) Endometrioid grades scores plot. (B) Non-endometrioid subtypes scores plot. (C) Endometrioid grades cluster vector plot (grade I as comparator). (D) Non-endometrioid subtypes cluster vector plot (uterine serous as comparator).

\section{Classification of tumour subtypes}

Tumours were subclassified according to their histological appearance. Endometrioid cancers were separated by grade and compared with one another (see Figure $5 \mathrm{~A}$ and $\mathrm{C}$ ), and the non-endometrioid cancers were compared separately (Figure 5B and D). Figure 5A shows separation between the three grades of endometrioid endometrial cancer. There are also regions of overlap between each grade and centrally between all three. Figure $5 \mathrm{C}$ compares the cluster vectors of grade 2 and grade 3 tumours against those of grade 1 tumours. Interestingly and surprisingly, grade 1 tumours appear more similar to grade 3 tumours than to grade 2 . The most notable peaks in the cluster vector plot for grade 3 tumours are between 1800 and $1450 \mathrm{~cm}^{-1}$, that is, in the lipid region and in Amide I/II.

Figure $5 \mathrm{~B}$ is a $3 \mathrm{D}$ scores plot, thus clustering and separation is dependent upon rotation. The clustered clear cell spectra separate clearly along LD1 from the amalgamated clusters of carcinosarcoma, uterine serous, and mixed spectra. The adenosarcoma spectra are dispersed between the two groupings. Along LD2, the uterine serous spectra separate from the carcinosarcoma, adenosarcoma, and clear cell spectra. LD3 separates the mixed group from all apart from adenosarcoma. When viewed in 3D, tight clusters are formed by the clear cell and carcinosarcoma groups. The uterine serous spectra are more spread out and overlap with the clear cell spectra. The mixed and adenosarcoma spectra, while still forming clusters, are more diffuse again and overlap with several other groups. Figure 5D compares uterine serous against all the other non-endometrioid tumour types. Adenosarcoma and carcinosarcoma appear alike in places; they have histological similarities as both contain glandular and connective tissue elements. Clear cell tumours are the most different from the other subtypes. Mixed tumours appear to have the most in common with uterine serous; as they often contain areas of uterine serous architecture, this is unsurprising.

\section{DISCUSSION}

This preliminary study demonstrates that ATR-FTIR spectroscopy has the potential to distinguish between benign and malig- nant endometrial tissues, and various subtypes thereof. It also appears capable of differentiating between normal tissues classified by phase of the menstrual cycle, although it was not able to distinguish between menstrual and proliferative tissues. This may be because the processing technique eliminates any loose surface shedding endometrium, revealing the very early (biochemically similar) proliferative tissue beneath. The most conspicuous differences are within the mid- and late-secretory phases, with a large accumulation of glycogen in the mid-secretory phase and rapid change in lipids in the late-secretory phase, together with structural protein changes indicated by several peaks in the Amide I/II areas. These changes are likely to be caused by preparation for the implantation of an embryo.

An important difference between tumour and benign tissue lies in the lipid area of the spectrum. The lipid absorbance in endometrioid cancers is greatest in grade 2 and 3 tumours, and among the non-endometrioid cancers in clear cell tumours, adenosarcomas and carcinosarcomas. This may represent qualitative or quantitative cell membrane changes. Further differences are present in the structural protein (Amide I/II) region. These are larger in endometrioid than non-endometrioid cancers, especially in Amide I. When tumours are compared with their corresponding tumour-adjacent tissue, a striking difference is observed between endometrioid and non-endometrioid cancers. In endometrioid cancer, the tumour-adjacent tissue almost resembles proliferative endometrium; in non-endometrioid cancer, it has more features in common with the adjacent cancer. This may be related to the pathogenesis of the two tumour types. Endometrioid cancer is strongly associated with unopposed oestrogen. This will produce proliferative changes in the non-cancerous adjacent tissue, perhaps too small to detect with histopathology, but discernable by spectroscopy.

Small areas of focal intraepithelial carcinoma in tumouradjacent tissue could explain its similarity to the corresponding non-endometrioid tumour. However, Figure 4E suggests a good degree of clustering of spectral points derived from tumouradjacent tissue pointing to this influence being minimal. Unfortunately, it was not possible to confirm this with conventional 
histopathology in the current study although all the tissue sections were $\mathrm{H} \& \mathrm{E}$-stained and examined by a clinical pathologist postspectroscopy. However, significant abnormality in the tumouradjacent tissue is unlikely; when benign and tumour-adjacent tissues were compared, the categories were remarkably similar in the resultant scores plot (Figure 4E). Endometrioid carcinomas can be separated by grade, although there is a region of overlap on the scatter plot. Clear cell tumours are spectrally different from the rest of the non-endometrioid cancer group. They have a distinctive molecular signature, which they share with ovarian and renal clear cell disease (Zorn et al, 2005). It is not known whether they also share spectral similarities.

As malignant endometrial tissue differs fundamentally from normal tissue in terms of structure, genetics, and cellular activity, ATR-FTIR spectroscopy followed by multivariate analysis should be capable of distinguishing between them. Our data show some overlap between spectra from different tissue types, potentially leading to misclassification. The reasons for this are both methodological and biological. The method of ATR-FTIR spectroscopy followed by PCA-LDA described here was initially developed to distinguish between grades of cervical cytology (Walsh et al, 2007) and has since been applied successfully to prostate tissue (Martin et al, 2007). Principal component analysisLDA is able to demonstrate data separation and highlight important wavenumbers; however, in its present form it is inadequate to predict the pathology of blindly presented samples. The total number of spectra obtained here $(n=1999)$ is good for a preliminary study. When broken down to individual pathological entities, the numbers are small and the results, while promising, should be interpreted with caution. Alternative mathematical models and data processing methods are available and when combined with large training data sets more successful predictive models will be developed (Kelly et al, 2010).

The tissue collection and processing methodology was designed to be simple and reproducible. While it succeeds in this, a major drawback is that it is impossible to entirely exclude myometrial contamination. As myometrial spectra are likely to be similar regardless of the endometrial histological pattern, this could account for some of the areas of overlap. In future, this could be overcome by modifying the method of sample collection. Using either a suction catheter (e.g., Pipelle) or an endometrial brush (e.g., Tao brush) sampling device would significantly reduce the chance of harvesting myometrial tissue. It would also allow tissue collection from women not undergoing hysterectomy and to eliminate the need for a histopathologist at the point of sample collection. However, this method could not have been used to compare tumour with tumour-adjacent tissue as has been demonstrated here.

In a biological context, the fact that perfect segregation of cluster categories is not observed is unsurprising, as one would expect some degree of overlap associated with progression or relatedness of different cell types. This is, in part, because living material is seldom uniform and endometrial tissue is no exception. Tumour tissue, especially from a small or low-grade cancer, may contain a significant proportion of normal cells. In addition, the tissue examined by the histopathologist may have a different pathological appearance from the tumour-adjacent tissue used for spectroscopy. It has been postulated that some high-grade tumours develop from low-grade lesions by accumulating oncogenic traits (Llobet et al, 2009). Tumours may therefore contain a mixed cell population within the high-grade lesion as it transforms from a low-grade precursor. The overlapping regions on the scores plots could be explained by a combination of the above factors.

The current gold standard in determining tumour type and grade is expert interpretation by a consultant histopathologist. Because of the complex and subjective nature of the task, opinions occasionally differ. Biospectroscopy is an emerging discipline that applies spectroscopic techniques developed by physical chemists to biological material. Rather than using structural differences, tissue is analysed at a molecular level and yields objective results. Such techniques are therefore ideal for gathering data complementary to the traditional histology described above. The precise pathological findings are pivotal in planning the treatment of patients with endometrial cancer and an objective test could have a valuable supplementary diagnostic role. This technique may be particularly suitable as a screening technique for endometrial biopsies, giving a yes/no/maybe to the presence of cancer, thus determining the need for further investigation. This could prove useful in resource-poor settings where expert pathological services are limited.

\section{ACKNOWLEDGEMENTS}

Rosemere Cancer Foundation supported this work. We thank theatre staff, nurses, porters, laboratory staff, and patients of Lancashire Teaching Hospitals NHS Trust who facilitated this study.

\section{REFERENCES}

Baker M, Gazi E, Brown M, Shanks J, Clarke N, Gardner P (2009) Investigating FTIR based histopathology for the diagnosis of prostate cancer. J Biophotonics 2: 104-113

Bokhman JV (1983) Two pathogenetic types of endometrial carcinoma. Gynecol Oncol 15: $10-17$

D’Angelo E, Prat J (2010) Uterine sarcomas: a review. Gynecol Oncol 116: $131-139$

Fadare O, Zheng W (2008) Endometrial glandular dysplasia (EmGD): morphologically and biologically distinctive putative precursor lesions of type II endometrial cancers. Diagn Pathol 3: 6

German MJ, Hammiche A, Ragavan N, Tobin MJ, Cooper LJ, Matanhelia SS, Hindley AC, Nicholson CM, Fullwood NJ, Pollock HM, Martin FL (2006) Infrared spectroscopy with multivariate analysis potentially facilitates the segregation of different types of prostate cell. Biophys $J$ 90: $3783-3795$

Horn L-C, Meinel A, Handzel R, Einenkel J (2007) Histopathology of endometrial hyperplasia and endometrial carcinoma: an update. Ann Diagn Pathol 11: 297-311

Kelly JG, Angelov PP, Trevisan J, Vlachopoulou A, Paraskevaidis E, Martin-Hirsch PL, Martin FL (2010) Robust classification of low-grade cervical cytology following analysis with ATR-FTIR spectroscopy and subsequent application of self-learning classifier eClass. Anal Bioanal Chem 398: $2191-2201$

Kelly JG, Singh MN, Stringfellow HF, Walsh MJ, Nicholson JM, Bahrami F, Ashton KM, Pitt MA, Martin-Hirsch PL, Martin FL (2009) Derivation of a subtype-specific biochemical signature of endometrial carcinoma using synchrotron-based Fourier-transform infrared microspectroscopy. Cancer Lett 274: $208-217$

Llabjani V, Trevisan J, Jones KC, Shore RF, Martin FL (2010) Binary mixture effects by PBDE congeners $(47,153,183$, or 209) and PCB congeners (126 or 153) in MCF-7 cells: biochemical alterations assessed by IR spectroscopy and multivariate analysis. Environ Sci Technol 44: $3992-3998$

Llobet D, Pallares J, Yeramian A, Santacana M, Eritja N, Velasco A, Dolcet X, Matias-Guiu X (2009) Molecular pathology of endometrial carcinoma: practical aspects from the diagnostic and therapeutic viewpoints. J Clin Pathol 62: 777-785

Martin FL, McLean AEM (1995) Adenosine triphosphate (ATP) levels in paracetamol-induced cell injury in the rat in vivo and in vitro. Toxicology 104: $91-97$

Martin FL, German MJ, Wit E, Fearn T, Ragavan N, Pollock HM (2007) Identifying variables responsible for clustering in discriminant analysis 
of data from infrared microspectroscopy of a biological sample. J Comput Biol 14: 1176-1184

Martin FL, Kelly JG, Llabjani V, Martin-Hirsch PL, Patel II, Trevisan J, Fullwood NJ, Walsh MJ (2010) Distinguishing cell types or populations based on the computational analysis of their infrared spectra. Nat Protoc 5: $1748-1760$

Maziak D, Do M, Shamji F, Sundaresan S, Perkins G, Wong P (2007) Fourier-transform infrared spectroscopic study of characteristic molecular structure in cancer cells of esophagus: an exploratory study. Cancer Detect Prev 31: 244-253

Renehan AG, Tyson M, Egger M, Heller RF, Zwalen M (2008) Body mass index and incidence of cancer: a systematic review and meta-analysis of prospective observational studies. Lancet 371: 569-579

Robles F, López de la Osa E, Lerner U, Johannisson E, Brenner P, Hagenfeldt K, Diczfalusy E (1972) $\alpha$-Amylase, glycogen synthetase and phosphorylase in the human endometrium: influence of the cycle and the $\mathrm{Cu}$-T device. Contraception 6: $373-384$

Sahu RK, Argov S, Walfisch S, Bogomolny E, Moreh R, Mordechai S (2010) Prediction potential of IR-micro spectroscopy for colon cancer relapse. Analyst 135: 538-544

Schindler AE (2009) Progestogen deficiency and endometrial cancer risk. Maturitas 62: $334-337$

Sherman ME (2000) Theories of endometrial carcinogenesis: a multidisciplinary approach. Mod Pathol 13: 295-308

Sindhuphak R, Issaravanich S, Udomprasertgul V, Srisookho P, Warakamin S, Sindhuphak S, Boonbundarlchai R, Dusitsin N (2003) A new approach for the detection of cervical cancer in Thai women. Gynecol Oncol 90: 10-14

Trevisan J, Angelov PP, Patel II, Najand GM, Cheung KT, Llabjani V, Pollock HM, Bruce SW, Pant K, Carmichael PL, Scott AD, Martin FL (2010) Syrian hamster embryo (SHE) assay ( $\mathrm{pH}$ 6.7) coupled with infrared spectroscopy and chemometrics towards toxicological assessment. Analyst 135: 3266-3272

Uharcek P (2008) Prognostic factors in endometrial carcinoma. J Obstet Gynaecol Res 34: $776-783$

Walsh MJ, Singh MN, Pollock HM, Cooper LJ, German MJ, Stringfellow HF, Fullwood NJ, Paraskevaidis E, Martin-Hirsch PL, Martin FL (2007) ATR microspectroscopy with multivariate analysis segregates grades of exfoliative cervical cytology. Biochem Biophys Res Commun 352: $213-219$

Walsh MJ, Singh MN, Stringfellow HF, Pollock HM, Hammiche A, Grude O, Fullwood NJ, Pitt MA, Martin-Hirsch PL, Martin FL (2008) FTIR microspectroscopy coupled with two-class discrimination segregates markers responsible for inter- and intra-category variance in exfoliative cervical cytology. Biomark Insights 3: 179-189

Yared E, Macmillan TJ, Martin FL (2002) Genotoxic effects of oestrogens in breast cells detected by the micronucleus assay and the Comet assay. Mutagenesis 17: 345-352

Zorn KK, Bonome T, Gangi L, Chandramouli GVR, Awtrey CS, Gardner GJ, Barrett JC, Boyd J, Birrer MJ (2005) Gene expression profiles of serous, endometrioid, and clear cell subtypes of ovarian and endometrial cancer. Clin Cancer Res 11: 6422-6430 